\title{
Sample-Selection Method for Arbitrary Fading Emulation Using Mode-Stirred Chambers
}

\author{
Juan D. Sánchez-Heredia, Mathias Grudén, Juan F. Valenzuela-Valdés, and \\ David A. Sánchez-Hernández, Senior Member, IEEE
}

\begin{abstract}
Mode-stirred chambers (MSCs) consist of one or more resonant cavities coupled in some way in order to allow the measurement of different antenna parameters such as antenna efficiency, correlation, diversity gain, or multiple-input-multiple-output (MIMO) capacity, among others. In a single-cavity MSC, also known as a reverberation chamber (RC), the environment is isotropic and the amplitude of the signal is Rayleigh distributed. Real environments, however, rarely follow an isotropic Rayleigh-fading scenario. Previous results have shown that a Rician-fading emulation can be obtained via hardware modification using an RC. The different methods lack from an accurate emulation performance and are strongly dependent upon chamber size and antenna configurations. With the innate complexity of more-than-one-cavity MSC, the coupling structure generates sample sets that are complex enough so as to contain different clusters with diverse fading characteristics. This letter presents a novel method to accurately emulate a more realistic Rician-fading distribution from a Rayleigh-fading distribution by selecting parts of the sample set that forms different statistical ensembles using a complex two-cavity multi-iris-coupled MSC. Sample selection is performed using a genetic algorithm. Results demonstrate the potential of MSCs for versatile MIMO fading emulation and over-the-air (OTA) testing. The method is patent protected by EMITE Ing., Murcia, Spain.
\end{abstract}

Index Terms-Diversity gain, mode-stirred chamber (MSC), multiple-input-multiple-output (MIMO) capacity.

\section{INTRODUCTION}

A RECENT technique to evaluate the multiple-input-multiple-output (MIMO) performance of a set of antennas is to measure them using a mode-stirred chamber (MSC). Unlike reverberation chambers (RCs), where only one cavity is used, MSCs may contain more than one metal cavity. Cavities are coupled by diverse means, and a rich fading environment consisting of diverse clusters with different fading characteristics can be obtained. The MSC also contains a set of mode stirrers that change the boundary conditions of the main cavity within

Manuscript received February 23, 2010; revised April 07, 2010; accepted April 19, 2010. Date of publication May 06, 2010; date of current version May 17, 2010. This work was supported in part by the Spanish National R\&D Programme through TEC2008-05811 and by Fundación Séneca, the R\&D coordinating agency for the Region of Murcia (Spain) under the 11783/PI/09 project.

J. D. Sánchez-Heredia and D. A. Sánchez-Hernández are with the Departamento de Tecnologías de la Información y Comunicaciones, Universidad Politécnica de Cartagena, Cartagena 30202, Spain (e-mail: jd.sanchez@ upct.es; david.sanchez@upct.es).

J. F. Valenzuela-Valdés is with EMITE Ingenieria, 30100 Murcia, Spain (e-mail: juan.valenzuela@emite-ingenieria.es).

M. Grudén is with Uppsala University, 75121 Uppsala, Sweden (e-mail: mathias.gruden@angstrom.uu.se).

Color versions of one or more of the figures in this letter are available online at http://ieeexplore.ieee.org.

Digital Object Identifier 10.1109/LAWP.2010.2049729 the chamber. This causes a multireflective environment that is repeatable and can be statistically studied. MSCs can be used as a very fast, easy, and accurate tool to measure a wide variety of MIMO parameters [1], [2]. There is yet a shortage of studies of their delay spread and isotropicity properties. In consequence, its potential ability to emulate realistic environments is normally put into jeopardy and typically degraded to that of simple single-cavity RCs. RCs typically provide a fading environment that is isotropic, and the amplitude of the signal is Rayleigh-distributed. Diverse isolated enhancements can be found in the literature for RCs, including the emulation of nonisotropic environments [3], indoor environments with different RMS delay spreads [4], wideband in-vehicle environments [5], keyhole effects [6], or metallic windows, trees, walls and other artifacts in buildings [7]. Yet, the assumptions were only valid for large chambers, and changes in the antenna configuration and/or in the chamber itself were required.

The use of multiple cavities on MSCs can provide for a more complicated fading emulation. With multiple cavities, MSCs can reduce the typically high-elevation angular spread of RCs, which approaches the emulated scenario to that typically encountered in a city street microcell when the mobile station gets farther away from the base station [8]. Thus, MSCs abandon the typical Clarke's model followed by single-cavity RCs. Rician-fading has also been emulated using RCs in [9] with hardware modifications. Since the coupling mechanisms in a multicavity MSC are complex and the sample set size is large, in this letter a novel method to emulate more realistic arbitrary-fading environments using a two-cavity MSC is presented. This is done by using a sample-selection technique. By keeping or discarding specific samples, it is possible to achieve a new subset with different fading distribution characteristics. The new subset consists only of unmodified measured samples forming a statistical ensemble different from Rayleigh. No hardware changes are required, neither in the chamber itself nor in the antenna configuration. The technique demonstrates the versatility of MSCs to emulate realistic MIMO scenarios. The method is patent-protected by EMITE Ing., Murcia, Spain.

\section{Measurement Setup}

Measurements illustrated in this letter have been performed with the E200 $8 \times 8$ MIMO Analyzer by EMITE Ing., in connection to the Rohde \& Schwarz ZVRE Vector Network Analyzer $(9 \mathrm{kHz}$ to $4 \mathrm{GHz})$. The E200 MIMO Analyzer is a MSC with dimensions of $0.82 \times 1.275 \times 1.95 \mathrm{~m}^{3}$, eight exciting antennas, polarization stirring due to aperture coupling and to the different orientation of the antenna exciting elements, three mechanical and mode-coupling stirrers, one 


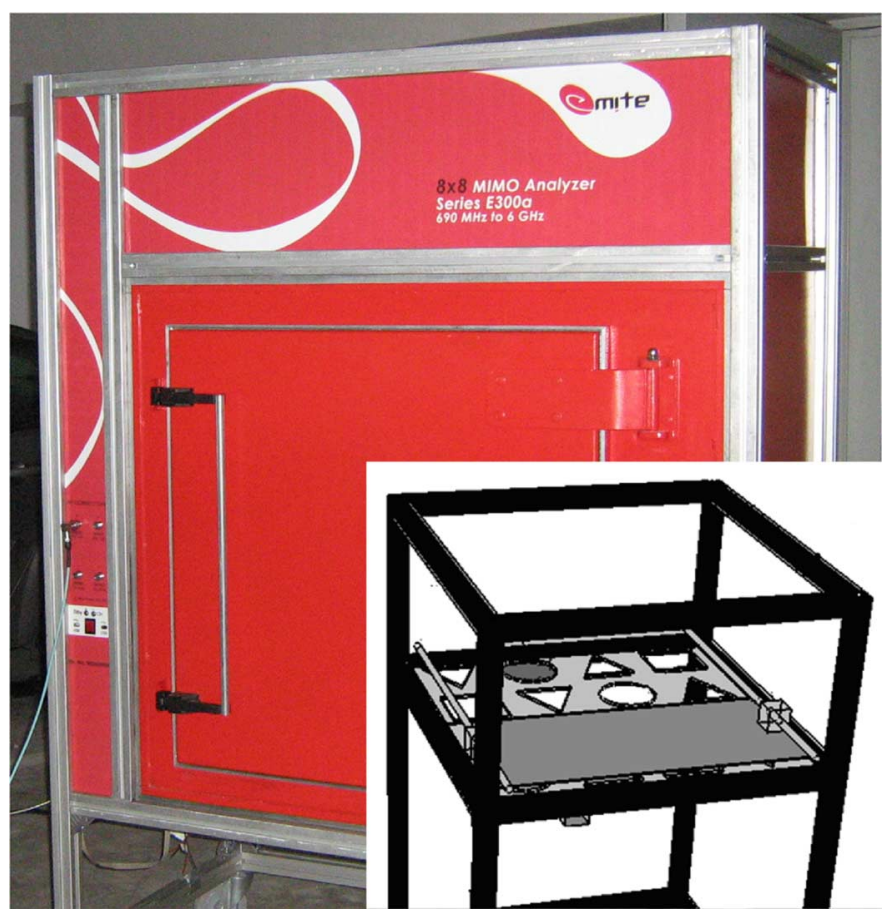

Fig. 1. A two-cavity mode-stirred chamber.

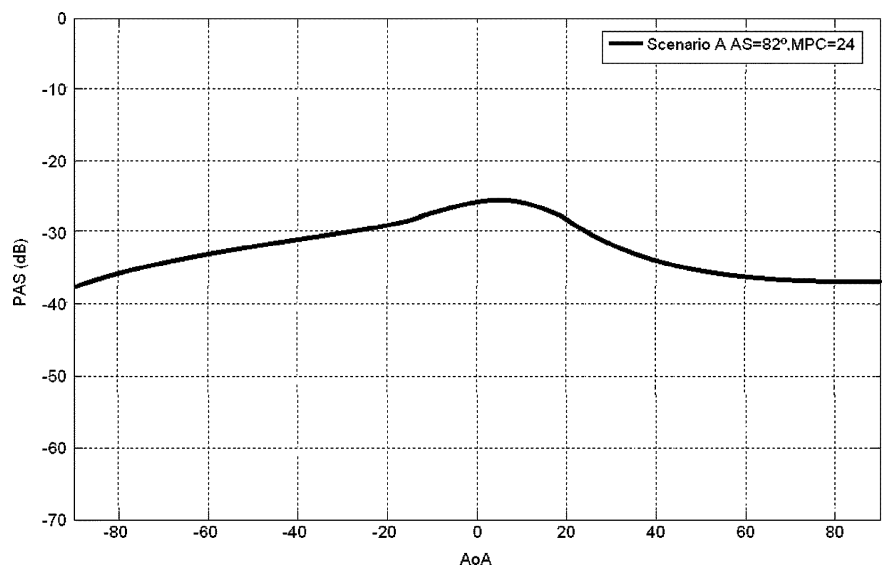

Fig. 2. Histogram of measured angle-of-arrival (AoA) using the high resolution MUSIC algorithm for the initial Rayleigh-fading measured scenario.

holder-stirrer, and variable iris-coupling. As depicted in Fig. 1, the E200 MIMO Analyzer is composed of two cavities that are coupled through a slotted metallic plate. Accurate control over the slots apertures allow for a complex coupling structure from the upper cavity, where the transmission antennas are placed, to the lower main cavity, wherein the MIMO device under test (DUT) is placed. The E200 MIMO Analyzer was set up for three holder positions with 15 different mechanical stirrer positions for each holder position, 12 iris-coupling aperture stirring, and $20 \mathrm{MHz}$ frequency stirring. Measurements were performed at $1800 \mathrm{MHz}$, and one half-wave dipole was used as the DUT located at the center of the chamber. The measured scenario " $\mathrm{A}$ " is an empty chamber with a typical quasi-isotropic behavior consisting of 50000 samples. The quasi-isotropic behavior of the measured scenario is reproduced in Fig. 2, wherein AS is defined as in [10].

\section{SAmple-Selection Technique}

The sample-selection technique consists of extracting the subset that conforms to a specific target fading statistical ensemble from the whole sample set measured in the E200. An algorithm has to decide whether each measured sample has to be kept or discarded. In this letter, an evolutionary method of a genetic algorithm (GA) [11] is employed to optimize the fitness between the density function of an ensemble of samples and a Rician-fading target density function that was a priori defined. GAs operate on a population of potential solutions applying the principle of survival of the fittest to produce successively better approximations to a solution. At each generation of a GA, a new set of approximations is created by the process of selecting individuals according to their level of fitness in the problem domain and reproducing them using operators borrowed from natural genetics. This process leads to the evolution of populations of individuals that are better suited to their environment than the individuals from which they were created, just as in natural adaptation. Inspired by the process of biological evolution, GA evolutionary methods employ stochastic search for obtaining the optimal solution. The key property of evolutionary methods is that occasionally very large changes in the solution are introduced. The presence of such large changes and random variations implies that evolutionary methods can find good solutions even in extremely complex discontinuous spaces or fitness landscapes that are hard to address by techniques such as gradient descent. This is why the GA-evolutionary algorithm was employed in this letter. The GA implemented in the Matlab toolbox gads was initially used. The Matlab-based GA allows for a combination of the innate advantages of GA optimization with all the functions implemented in all the other toolboxes of this software. The application of GAs to the sample-selection problem consists of assigning one binary variable ( 0 or 1 ) to each sample, as it happens in the typical GA binary string called "chromosome," representing whether this sample will be in the final solution or not. The GA allows for a bit string to embrace the final solution, which can be quickly obtained from the chromosome population of the genetic algorithm.

With an initial Rayleigh-fading distribution from the complete sample set typically emulated by an MSC, the error in the achieved target Rician distribution is analyzed by calculating the mean-square error between the achieved and the target distributions as

$$
\varepsilon_{d}=\frac{1}{Q} \sum_{x=0}^{Q}\left(f(x)-f_{\text {target }}(x)\right)^{2} d x
$$

where $Q$ is the number of elements in the array, $d x$ is the distance between two $x$-elements in the probability density function (PDF), $f(x)$ is the Rician-fading target distribution, and $f_{\text {target }}(x)$ is the achieved target distribution with a subset of measured samples. In addition to $\varepsilon_{d}$, the relative amount of discarded data is noted since it will affect the final accuracy. The employed error (fitness) function within the GA was a sum squared error (SSE)-based function, which can be easily implemented at a reasonable computation complexity for the task at hand. Since, for the sample-selection problem, we are looking 


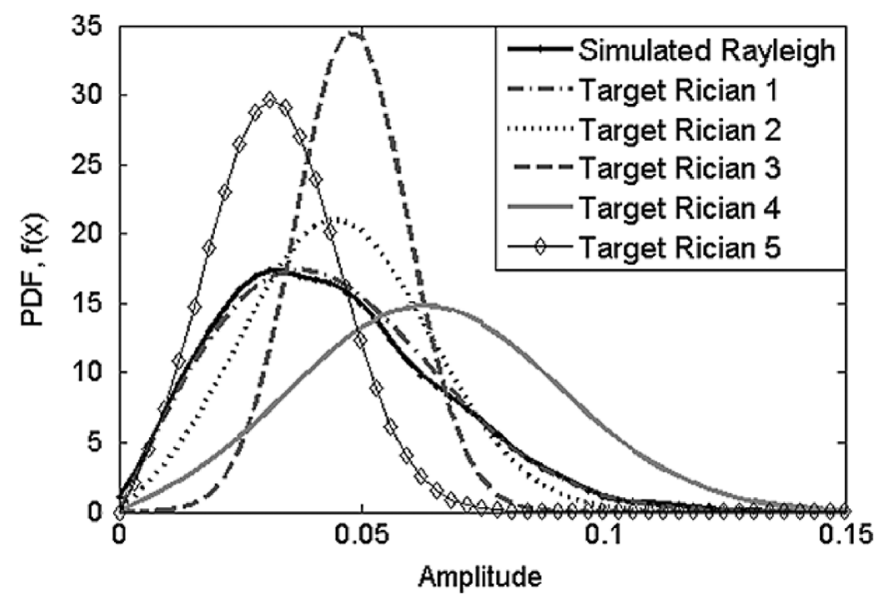

Fig. 3. Initial (ideal theoretical Rayleigh) and target (ideal theoretical Rician) fading distributions.

for best fits to target density functions, which range from 0 to 1 , the conventional SSE formula was modified as

$$
\operatorname{error}_{i}=\sum_{i=1}^{n}\left[f_{i}(n)-f_{\text {target }}(n)\right]^{2} / \sum_{i=1}^{n}\left[f_{\text {target }}(n)\right]^{2}
$$

where $n$ is the number of bins used to calculate the density function. To achieve a reasonable ratio between computing time and accuracy, the fitness value was limited to 0.01 by default. Since the phase distribution in an RC is typically uniform over $2 \pi$, in this letter the goal includes maintenance of this phase distribution on the final subset. In a more general case also, the phase of the data can be included in the PDF. If both the initial and the target distribution are assumed to be Rician-fading, for a complex sample

$$
z=x+i y
$$

the PDFs of the initial and final distributions in the complex plane are

$$
\begin{aligned}
& f_{\text {initial,compl }}(x, y) \\
& =\frac{x y}{s_{\text {initial }}^{4}} \exp \left(-\frac{x^{2}+a_{x, \text { initial }}^{2}+y^{2}+a_{y, \text { initial }}^{2}}{2 s_{\text {initial }}^{2}}\right) \\
& \quad \cdot I_{0}\left(\frac{x a_{x, \text { initial }}}{s_{\text {initial }}^{2}}\right) \cdot I_{0}\left(\frac{y a_{y, \text { initial }}}{s_{\text {initial }}^{2}}\right) \\
& \left.f_{\text {target,compl }} x, y\right) \\
& =\frac{x y}{s_{\text {target }}^{4}} \exp \left(-\frac{x^{2}+a_{x, \text { target }}^{2}+y^{2}+a_{y, \text { target }}^{2}}{2 s_{\text {target }}^{2}}\right) \\
& \cdot I_{0}\left(\frac{x a_{x, \text { target }}}{s_{\text {target }}^{2}}\right) \cdot I_{0}\left(\frac{y a_{y, \text { target }}}{s_{\text {target }}^{2}}\right) .
\end{aligned}
$$

\section{SAMPLE-SELECtion Performance}

For comparison purposes, the performance analysis is performed by treating both a set of 10000 scalar ideal Rayleigh distributed data samples from theoretical formulas and the 50000 sample set measured at scenario "A" as illustrated in Fig. 2. Five different Rician-fading target distributions were defined and are depicted in Fig. 3. The specific parameters for each distribution
TABLE I

TARGET RICIAN-FADING DISTRIBUTIONS

\begin{tabular}{|c|c|c|c|c|}
\hline $\begin{array}{c}\text { Target } \\
\text { number }\end{array}$ & $\mathrm{a}_{2}$ & $\mathrm{~s}_{2}$ & $\begin{array}{c}\text { K-factor } \\
{[\text { linear] }}\end{array}$ & $\mathrm{P}_{\text {out }} / \mathrm{P}_{\text {in }}$ \\
\hline Rician 1 & 0.0286 & 0.0286 & 0.5 & 1 \\
\hline Rician 2 & 0.0404 & 0.0202 & 2 & 1 \\
\hline Rician 3 & 0.0467 & 0.0117 & 8 & 1 \\
\hline Rician 4 & 0.0572 & 0.0286 & 2 & 2 \\
\hline Rician 5 & 0.0286 & 0.0143 & 2 & 0.5 \\
\hline
\end{tabular}

TABLE II

Performance of SAmple-Selection Technique With GA

\begin{tabular}{|c|c|c|c|c|}
\hline $\begin{array}{c}\text { Initial sample } \\
\text { set }\end{array}$ & $\begin{array}{c}\text { Target } \\
{\left[\varepsilon_{d}\right]}\end{array}$ & $\begin{array}{c}\text { Error } \\
{\left[\varepsilon_{d}\right]}\end{array}$ & $\begin{array}{c}\text { Discarded } \\
\text { samples } \\
{[\%]}\end{array}$ & $\begin{array}{c}\text { Computation } \\
\text { time } \\
{[\mathrm{s}]}\end{array}$ \\
\hline Id. Rayleigh & Rician 1 & 0.0011 & 55.1 & 1.6 \\
\hline Id. Rayleigh & Rician 2 & 0.0008 & 51.3 & 62 \\
\hline Id. Rayleigh & Rician 3 & 0.0012 & 60.8 & 1168 \\
\hline Id. Rayleigh & Rician 4 & 0.0005 & 70.3 & 1622 \\
\hline Id. Rayleigh & Rician 5 & 0.0011 & 55.2 & 516 \\
\hline Meas.Rayleigh & Rician 1 & 0.0013 & 50.7 & 102 \\
\hline Meas.Rayleigh & Rician 2 & 0.0015 & 53.7 & 724 \\
\hline Meas.Rayleigh & Rician 3 & 0.0024 & 67.2 & 5225 \\
\hline Meas.Rayleigh & Rician 4 & 0.0011 & 75.5 & 7948 \\
\hline Meas.Rayleigh & Rician 5 & 0.0021 & 54.6 & 1030 \\
\hline
\end{tabular}

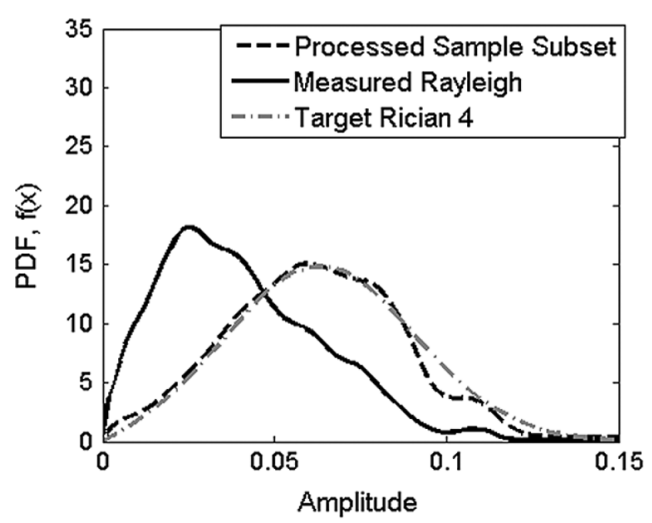

Fig. 4. A comparison between initial measured Rayleigh-fading set, processed sample subset, and Rician-fading target 4.

are also illustrated in Table I. Three of the distributions have the same mean output power as the initial ideal Rayleigh-fading distribution. The other two have a larger and lesser output power, respectively.

The measured figures of merit are depicted in Table II. From this table, it can be observed that very accurate Rician-fading emulation can be achieved with the sample-selection technique. It is also noticeable from this table that a large percentage of samples is discarded when distributions with larger mean output power than originally measured ones are targeted. The GA seems to discard more samples for those target distributions that have a larger output power than the initial Rayleigh-fading set, which poses a limitation to the technique. A comparison between Rician-fading target 4 and measured subset extracted using the sample-selection technique is illustrated in Fig. 4 as an example. 
It can also be observed from this figure that the use of large 50000 -samples measured Rayleigh-fading data makes the GA spend more time, which is to be expected due to the larger number of initial samples. Using both theoretical and measured Rayleigh-fading, initial sample sets provide very similar accuracies on final sample subsets. Likewise, it seems clear from the results that the higher the K-factor of the target Rician distribution, the higher the required computational time of the GA.

\section{CONCLUSION}

With the sample-selection technique, this letter has demonstrated the ability of MSCs to accurately emulate Rician-fading distributions. This has been done without any hardware alteration within the MSC for the first time. The obtained data set is composed of unmodified measured samples conforming a Rician-fading statistical ensemble. The sample-selection technique can further enhance the capabilities of MSCs for MIMO OTA measurements, closing the current gap between MSCs and more expensive spatial-fading emulators. Future research includes the extension to more general arbitrary-fading distributions, the optimization and/or hybridization of the GA so as to be able to compute real-time for compliance-testing purposes, and the calculation and analyses of novel MIMO capacity and diversity gain curves through the use of the processed data subsets. The technique is patent-protected by EMITE Ing.

\section{REFERENCES}

[1] P. Hallbjorner and K. Madsen, "Terminal antenna diversity characterisation using mode stirred chamber," Electron. Lett., vol. 37, no. 5, pp. 273-274, Mar. 2001.

[2] J. F. Valenzuela-Valdés et al., "Evaluation of true polarization diversity for MIMO systems," IEEE Trans. Antennas Propag., vol. 57, no. 9, pp. 2746-2755, Sep. 2009.

[3] J. F. Valenzuela-Valdés et al., "Emulation of MIMO non-isotropic fading environments with reverberation chambers," IEEE Antennas Wireless Propag. Lett., vol. 7, pp. 325-328, 2008.

[4] O. Delangre et al., "Wideband analysis of coupled reverberation chambers for testing MIMO systems," in Proc. IEEE PIMRC, Sep. 2006.

[5] O. Delangre et al., "Modeling in-vehicle wideband wireless channels using reverberation chamber theory," in Proc. IEEE Veh. Technol. Conf., Sep. 2007, pp. 2149-2153.

[6] O. Delangre et al., "Propagation channel modelling in coupled reverberation chambers for testing MIMO systems," in COST $273 \mathrm{TD}(05)$ 85, Jun. 2005.

[7] Z. Yun and M. F. Iskander, "MIMO capacity for realistic wireless communications environments," in Proc. IEEE Antennas Propag. Soc. Int. Symp., Jun. 2004, pp. 1231-1234.

[8] M. H. El-Sallabi et al., "Impacts of environment and antenna heights on angular spread of radiowave propagation in a city street microcell," in Proc. IEEE Antennas Propag. Soc. Int. Symp., 2000, pp. 1146-1149.

[9] C. L. Holloway et al., "On the use of reverberation chambers to simulate a Rician radio environment for the testing of wireless devices," IEEE Trans. Antennas Propag., vol. 54, no. 11, pp. 3167-3177, Nov. 2006.

[10] B. H. Fleury, "First- and second-order characterization of direction dispersion and space selectivity in the radio channel," IEEE Trans. Inf. Theory, vol. 46, no. 6, pp. 2027-2044, Sep. 2000.

[11] R. O. Duda, P. E. Hart, and D. G. Stork, Pattern Classification, 2nd ed. New York: Wiley, 2001. 